\title{
MRS and DTI Study in Cognitive Deficit in Patients with Type 2
} Diabetes Mellitus

\author{
Lan-mei CHEN, Yuan XU, Hai-du ZHANG and Wen-bin ZHENG ${ }^{a}{ }^{,}$ \\ Second Affiliated Hospital of Shantou University Medical College, Guangdong, China \\ ahwenb@126.com \\ ${ }^{*}$ Corresponding author
}

Keywords: Diabetes type 2(DM2), Cognitive function, Diffusion tensor imaging (DTI), $1 \mathrm{H}$ magnetic resonance spectroscopy (MRS).

\begin{abstract}
To investigate the cognitive function in patients with type 2 diabetes mellitus (T2DM) by $1 \mathrm{H}$ magnetic resonance spectroscopy (MRS) and diffusion tensor imaging (DTI). We studied forty-one patients with T2DM and the other group of thirty-five healthy control subjects by MRS and DTI. A region of interest (ROI) was positioned in the white matter of the centrum semiovale. The results indicated that the mean of the ratio of NAA to $\mathrm{Cr}(\mathrm{NAA} / \mathrm{Cr})$, the ratio of NAA to Cho(NAA/Cho), and the FA values decreased, while the ADC values increased in the diabetic patients $(P<0.01$, all $)$. Both of the mean in NAA/Cr and the ratio of $\mathrm{Cho}$ to $\mathrm{Cr}(\mathrm{Cho} / \mathrm{Cr})$ showed strong correlation to FA values in diabetic patients ( $r=0.603$ and $r=-0.646 ; P<0.01$, both). The FA values also correlated significantly with Mini-Mental State Examination (MMSE) ( $r=0.568$; $P<0.01)$. The relationship of Cho and NAA to the FA values, and thus to the content of neuronal structures in patients with T2DM, suggests that these metabolic changes are consistent with axonal loss or dysfunction. Diffusion tensor measurement is more effectively in monitoring the progression of the cognitive impairment in diabetic.
\end{abstract}

\section{Introduction}

Type 2 diabetes is characterized by the body's inability to control high blood glucose levels. Its complications include pathological changes of both small and large vessels, cranial, and peripheral nerves. The alteration in cognitive functioning is a clinical manifestation of cerebrovascular disease, which is a main cause of mild cognitive impairment in the elderly. Diabetes is one of the risk factor for dementia in both vascular type and Alzheimer's disease (AD). Patients with type 2 diabetes also have cognitive impairment. Patients with DM2 are more prone to brain atrophy, and they also have an increased risk for vascular lesions such as white matter lesions (WML) and infarcts [1]. Vascular lesions are associated with impaired cognitive functioning in patients with DM2 [2,3]. Using brain imaging technique may elucidate some of the mechanisms underlying cognitive decline in DM2. Diffusion tensor imaging (DTI) is a promising method for characterizing microstructural changes or differences with neuropathology. In addition, it also provides information on white matter tract integrity [4]. DTI measures the mean diffusivity [MD], which is a directionally averaged measure of diffusion, and the fractional anisotropy, which FA is a measure of the directionality of diffusion. Recent studies discovered that the DTI parameters were associated with the cognition of patients with ischaemic leukoaraiosis [5]. Magnetic resonance spectroscopy (MRS) provides a sensitive and noninvasive assessment of metabolic changes within the brain. MRS can obtain measurement of $\mathrm{N}$ acetylaspartate (NAA), a marker of neuronal integrity, which is associated with neuronal or axonal loss and damage [6]. An increase in choline (CHO) levels, which is involved in membrane synthesis and degradation, were found in acute demyelization [7].Combining DTI with MRS will reflect neuronal or axonal loss and damage. It can also measure the concentrations of cerebral metabolites. In this study, we investigated the relationship between DTI and MRS with cognitive impairment in elderly patients that were diagnosed with type 2 diabetes. Our hope for this study is 
to prove that both DTI and MRS play an important role in detecting the mild cognitive impairment early in patients that were diagnosed with type 2 diabetes.

\section{Methods}

\section{Subjects}

There were two groups of subjects for this study. The first group consists of forty-one patients (between the ages of 45-70 years old) with type 2 diabetes were admitted into the University Hospital. The criteria used for type 2 diabetes for this group is in accordance with the American Diabetes Association (ADA) [8]. The second group consists of thirty-five healthy control subjects that were not diabetic; whose glucose levels do not meet criteria for diabetes (between the ages of 45-65 years old). All participants' cognitive performances were assessed using Mini-Mental State Examination (MMSE), made by Folstein et al in 1975[9]. MMSE is the most influential cognitive function assessment tool, widely used at home and abroad because of its sensitivity and easy to operate. The scores of six domains from MMSE were recognized as the six cognitive function status of orientation, memory, language, visuospatial abilities, attention, and executive function separately. Subjects who lost more than 3 points at MMSE were categorized as having cognitive deterioration.

\section{Scan Acquisition}

All imaging were performed on a 1.5 T GE Signa MR scanner. A standard quadrature head coil was used for radiofrequency transmission and reception of the MR signal. Conventional MR imaging consisted of four sequences with slice locations covering the whole brain: T1weighted-repetition time (TR) ms/echo time (TE) $\mathrm{ms}=1800 / 28.3$; matrix size $228 \times 224$; FOV $24 \times 18$; thickness $=7 \mathrm{~mm}$. T2-weighted-TR/TE=3560/106; matrix size $384 \times 256$; FOV $24 \times 18$; thickness $=7 \mathrm{~mm}$. Fluid-attenuated inversion recovery (FLAIR)-TR/TE=9000/120; inversion time $2200 \mathrm{~ms}$; thickness $=7 \mathrm{~mm}$.

For proton MR spectroscopic imaging, a region of interest (ROI) for the supraventricular volume of $13 \times 13 \times 2 \mathrm{~cm}^{3}$ (210 voxels) was positioned in the center of an axial T2weighted(Figure.a); scout image was positioned above the ventricles through the centrum semiovale white matter, which is parallel to the canthomeatal line. Chemical shift imaging (CSI) was performed using the PRESS sequence with field of TR/TE 1500/135, Phase18, NEX 1, and one average. DTI were performed with a single-shot spin-echo echo-planar pulse sequence that used 25 diffusion-encoding directions. The minimum b-value was $0 \mathrm{~s} / \mathrm{mm}^{2}$; the maximum was $1000 \mathrm{~s} / \mathrm{mm}^{2}$. Section thickness $=5 \mathrm{~mm}$, and spacing $=0$, and the images were acquired with fat saturation.

\section{Data Processing}

Spectroscopic data was transferred to AW 4.5(GE Medical system, advantage window 4.5) workstation and processed using Functoo1 software. The common way to analyze clinical spectra is to look at metabolite ratios, namely NAA/Cr, NAA/Cho, and $\mathrm{Cho} / \mathrm{Cr}$. Peak area ratios of NAA/Cr, NAA/Cho, and Cho/Cr were obtained for each voxel. Chemical shift artefact causes a displacement of the excited volume for different metabolites. Metabolite ratios, from every acceptable spectrum, were collected for the entire transverse section for each subject. Voxels that were at the midline, which was due to CSF and grey matter contamination, were at the boundaries of the grid due to the effects of chemical shift artefact exclusion.

For DTI analysis, the data was also transferred to AW4.5 (GE Medical system advantage window 4.5) workstation and processed using Functool software. The DTI voxels were then matched to the MRS voxels and DTI software was used to obtain the ADC and FA values. CSF voxels were excluded in order to ensure CSF contamination of fractional anisotropy and mean diffusivity characteristics in the spectroscopic ROI were minimal. 


\section{Data Analysis}

Statistical analysis was performed by SPSS 19.0 software. Statistical comparisons of data groups were performed by using the two-tailed Student's t test. Pearson's correlation coefficients were used to evaluate the strength of the associations between the imaging, cognitive, and clinical variables. $P<0.05$ is considered as statistically significant.

\section{Results}

The MMSE in diabetic patients $(n=41)$ were lower than that in the healthy controls $(n=35) .(P<0.01$, Table 1). The CSI measurements are illustrated by Figure. a, b, c. For the main metabolite values (NAA/Cr, NAA/ Cho and Cho/Cr), and the ADC and FA values, are listed in Table 2. The metabolite ratios are introduced in Table 3. Compared with the controls, the mean FA value and the mean of NAA/Cr, NAA/Cho were significantly reduced in diabetic patients, and the ADC increased significantly $(\mathrm{P}<0.01$, both).

Table 1 MMSE in diabetes patients and in healthy controls

\begin{tabular}{ccc}
\hline & Diabetes patients & Controls \\
\hline MMSE & $25.804 \pm 1.981 * *$ & $29.55 \pm 0.550$ \\
\hline
\end{tabular}

Means \pm intersubject standard deviation (SD); ** $P<0.01$

Table 2 DTI and MRS derived values in diabetes patients and in healthy controls

\begin{tabular}{lccccc}
\hline & FA & ADC & Mean NAA/Cr & Mean NAA/Cho & Mean Cho/Cr \\
\hline $\begin{array}{l}\text { Diabetes } \\
\text { Patients }\end{array}$ & $0.401 \pm 0.141 * *$ & $7.93 \pm 0.954 * *$ & $1.790 \pm 0.223 * *$ & $1.682 \pm 0.284 * *$ & $1.129 \pm 0.143$ \\
\hline Controls & $0.434 \pm 0.113$ & $7.19 \pm 0.771$ & $2.089 \pm 0.392$ & $2.155 \pm 0.275$ & $0.986 \pm 0.140$ \\
\hline
\end{tabular}

FA fractional anisotropy, ADC apparent diffusion coefficient, Cho choline, Cr creatine, NAA N-acetyl aspartate;

$* P<0.05, * * P<0.01$

Table 3 Metabolite Ratios

\begin{tabular}{lccc}
\hline & Mean NAA/Cr & Mean NAA/Cho & Mean Cho/Cr \\
\hline Normal & 2.0 & 1.6 & 1.2 \\
\hline Abnormal & $<1.6$ & $<1.2$ & $>1.5$ \\
\hline
\end{tabular}

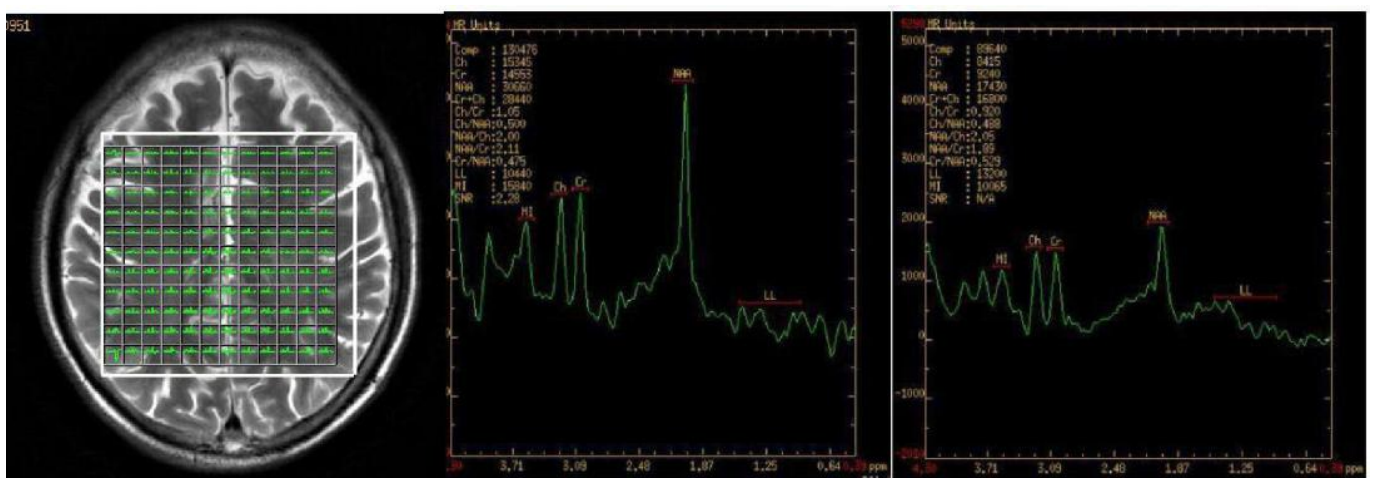

a

$\mathrm{b}$

$\mathrm{c}$

Figure. a) Axial T2-weighted image. The white line represents the region of interest selected for Spectroscopy. The green grid represents the position of individual voxels within the ROI. b) spectra of the corresponding voxels in a control .c) the corresponding spectra in a diabetes patient.

Table 4 shows the correlation (r) between DTI parameters (FA, ADC) and the 1H-MRS parameters (NAA/Cr, NAA/Cho and Cho/Cr). The positive correlation of the FA with the mean of NAA/Cr was significant, both in the diabetic patients and the controls $(r=0.603$ and $r=0.420$; $P<0.01)$. The diabetic patients showed significant negative correlations of the $\mathrm{FA}$ with $\mathrm{Cho} / \mathrm{Cr}$ 
( $r=-0.646 ; P<0.01$ ); however, the other correlation coefficients in Table 4 were not significant. In the controls, there was a positive correlation between the mean $\mathrm{Cho} / \mathrm{Cr}$ and $\mathrm{FA}(r=0.704 ; P<0.01)$, and a negative correlation with $\operatorname{ADC}(r=-0.655 ; P<0.01)$. There was also a negative correlation between FA and NAA/Cho $(r=-0.450 ; P<0.01)$, and a positive correlation between ADC and NAA/Cho $(r=0.383 ; P<0.01)$ in the controls.

Table 4 Correlations between mean DTI and mean MRS parameters in diabetes patients and in healthy controls

\begin{tabular}{lcccccccc}
\hline & \multicolumn{3}{c}{ Fractional anisotropy(FA) } & \multicolumn{3}{c}{ Mean diffusivity(ADC) } \\
& \multicolumn{2}{c}{ Diabetes patients } & Controls & Diabetes patients & \multicolumn{2}{c}{ Controls } \\
\cline { 2 - 9 } & $r$ & $p$ & $r$ & $p$ & $r$ & $p$ & $r$ & $p$ \\
\hline Mean NAA/Cr & 0.603 & $<0.01$ & 0.420 & $<0.01$ & - & $>0.05$ & 0.397 & $<0.01$ \\
Mean NAA/Ch & - & $>0.05$ & -0.405 & $<0.01$ & - & $>0.05$ & 0.383 & $<0.01$ \\
Mean Cho/Cr & -0.646 & $<0.01$ & 0.704 & $<0.01$ & - & $>0.05$ & -0.655 & $<0.01$ \\
\hline
\end{tabular}

In diabetic patients, the positive correlation between MMSE and FA was significantly, shown in Table 5( $r=0.568 ; P<0.01)$; however, the other correlation coefficients in Table 5 were only trends.

Table 5 the correlations between MMSE with both DTI and MRS parameters in diabetes patients

\begin{tabular}{ccccccccc}
\hline & \multicolumn{3}{c}{ FA } & \multicolumn{2}{c}{ ADC } & \multicolumn{2}{c}{ NAA/Cr } & \multicolumn{2}{c}{ Cho/Cr } \\
$r$ & $p$ & $r$ & $p$ & $r$ & $p$ & $r$ & $p$ \\
\hline 0.568 & $<0.01$ & 0.343 & $>0.05$ & 0.477 & $>0.05$ & 0.52 & $>0.05$ \\
\hline
\end{tabular}

\section{Discussion}

Recent studies (Manschot SM, 2006; Van Harten B, 2007) provided evidence that diabetes is associated with impaired cognitive processes in older adults, which may hasten cognitive decline and lead to dementia [2,3]. Henceforth, the presence of DM2 in patients with symptomatic arterial disease is related to cognitive dysfunction.

DTI is sensitive to tissue damage in cerebral small vessel disease, and it detects a characteristic reduction in fractional anisotropy and increase in mean diffusivity. These changes appear to be a good marker of disease burden and correlate better with cognition [4]. Pathological studies have reported axonal loss, ischaemic demyelination, loss of oligodendrocytes and gliosis in areas of leukoaraiosis is the primary process $[10,11,12,13]$, which would explain much of the pattern of DTI abnormalities reported.

Several recent studies discovered that diabetes can cause DNA fragmentation of hippocampal neurons, which can lead to neuronal degeneration, and effect the synaptic plasticity of hippocampus. Gold SM, et al. provided evidence of hippocampal damage (volume losses and impairments in memory and learning) in type 2 diabetes. This may provide a window into very early manifestations of brain complications in type 2 diabetes [14].

In our study, firstly, compared with normal controls, a reduction in FA and an increase in ADC in patients with diabetes mellitus were found. There is a positive correlation between cognition and the FA values in centrum semiovale where the axonal part of neurons is present. Secondly, we found a positive significant correlation between fractional anisotropy and the NAA/Cr ratio. The level of NAA, a compound present in neurons, known to protect against glutamate neurotoxicity, though its function in the neuron is still uncertain, is related to the extent of neuron structure in a given brain volume. These findings provide further evidence that axonal loss or dysfunction, or both, account for the FA abnormalities found in diabetic patients. It provides further evidence that DTI is identifying white matter tractdisruption, and therefore disconnection of cortical-cortical and subcortical-cortical circuits. 
Thirdly, we found a negative correlation between the Cho/Cr ratio and frctional anisotropy, in contrast to association with NAA/Cr. Previous reports showed that Cho increases as it is observed in a demyelinating lesion mode [15] and in normal appearing white matter of multiple sclerosis patients[16], which were associated with demyelination and the subsequent breakdown of neurons. Cho takes part in the composition of cell membrane and the formation of myelin. Myelin is considered as a maker to reflect the cell membrane phospholipids. Cell membrane collapse can increase the concentration of Choline, which may reflect the severity of inflammation, Wellen KE et al [17]. Acute demyelination has been associated with a rise of choline [18], and demyelization has also been reported as a histological feature of ischaemic leukoaraiosis [19]. Correlation between DTI and MRS parameters were present in patients with cerebral small vessel disease, primary progressive multiple sclerosis and adrenoleukodystrophy [20]. This finding suggests that these metabolic changes consistent with axonal loss or dysfunction.

\section{Conclusion}

Our results suggest that the increase in mean diffusivity, and decrease in fractional anisotropy found in patients with Diabetes Mellitus, is associated with metabolic changes and cognitive dysfunction. Diffusion tensor measurements are more effectively in monitoring the progression of the cognitive impairment in diabetes patients.

\section{Acknowledgements}

This research was financially supported by Medical Scientific Research Foundation of Guangdong Province, China (grant A2015541), Science and Technology Planning Project of Shantou City, China (grant No.2014242), Shantou, China.

\section{References}

[1] Van Harten B, de Leeuw FE, Weinstein HC, Scheltens P, Biessels GJ.Brain imaging in patients with diabetes: a systematic review. Diabetes Care .2006; 29(11):2539-48.

[2] Manschot SM, Brands AM, van der Grond J, Kessels RP, Algra A,Kappelle LJ,et al.Brain magnetic resonance imaging correlates of impaired cognition in patients with type 2 diabetes.Diabetes. 2006; 55(4):1106-13.

[3] Van Harten B, Oosterman J, Muslimovic D, van Loon BJ, Scheltens P, Weinstein HC.Cognitive impairment and MRI correlates in the elderly patients with type 2 diabetes mellitus. Age Ageing. 2007; 36(2):164-70.

[4] O' Sullivan M, Morris RG, Huckstep B, Jones DK, Williams SCR, Markus HS.Diffusion tensor MRI correlates with executive dysfunction in patients with ischaemic leukoaraiosis. J. Neurol. Neurosurg. Psychiatr. 2004; 75(3): 441-447.

[5] Basser PJ, Pierpaoli C. Microstructural and physiological features of tissues elucidated by quantitative-diffusion-tensor MRI. J. Magn. Resonan. B 1996; 111(3):209-219.

[6] Barker PB. N-acetyl aspartate - a neuronal marker? Ann. Neurol.2001; 49(4):423-424.

[7] Arnold DL, Matthews PM, Francis GS, O ' Connor J, Antel JP.Proton magnetic resonance spectroscopic imaging for metabolic characterization of demyelinating plaques. Ann. Neurol. 1992; 31(3): 235-241.

[8] American Diabetes Association. Diagnosis and classification of diabetes mellitus.Diabetes Care, 2010; 33(Supplement 1) 
[9] Folstein MF, Folstein SE, McHugh PR (). ""Mini-mental state". A practical method for grading the cognitive state of patients for the clinician". Journal of psychiatric research. 1975; 12 (3): 189-98.

[10] Revesz T, Hawkins CP, du Boulay EP, Barnard RO, McDonald WI.Pathological findings correlated with magnetic resonance imaging in subcortical arteriosclerotic encephalopathy (Binswanger's disease).J. Neurol. Neurosurg. Psychiatr. 1989; 52(12): 1337-1344.

[11] Van Swieten J, van den Hout J, van Ketel B, Hijdra A,Wokke J, van Gijn J. Periventricular lesions in the white matter on magnetic resonance imaging in the elderly. A morphometric correlation with arteriolosclerosis and dilated perivascular spaces. Brain 1991; 114(2): 761-774.

[12] Fazekas F, Kleinert R, Offenbacher H, Schmidt R, Kleinert G, Payer F, et al. Pathologic correlates of incidental MRI white matter signal hyperintensities. Neurology 1993; 43(9): 16831689.

[13] Moody DM, Thore CR, Anstrom JA, Challa VR, Langefeld CD, Brown WR. Quantification of afferent vessels shows reduced brain vascular density in subjects with leukoaraiosis. Radiology 2004; 233(3): 883-890.

[14] Gold SM, Dziobek I, Sweat V, et al. Hippocampal damage and memory impairments as possible early brain complications of type 2 diabetes.Diabetologia, 2007; 50(4): 711-719.

[15] Degaonkar MN, Khubchandhani M, Dhawan JK, Jayasundar R, Jagannathan NR. Sequential proton MRS study of brain metabolite changes monitored during a complete pathological cycle of demyelination and remyelination in lysophosphatidyl choline (LPC)-induced experimental demyelinating lesion model. NMR Biomed. 2002; 15: 293-300.

[16] Tartaglia MC, Narayanan S, De Stefano N, Arnaoutelis R, Anetel SB,Francis SJ, Santos AC, Lapierre Y, Arnold DL. Choline is increased in prelesional normal apppearring white matter in multiple sclerosis. J Neurol. 2002; 249: 1382-1390

[17] Wellen KE, Hotamisligil GS. Inflammation, stress, and diabetes. J Clin Invest, 2005; 115(5): 1111-1119.

[18] Barker PB, Breiter SN, Soher BJ, Chatham JC, Forder JR, Samphilipo MA, et al. Quantitative proton spectroscopy of canine brain: in vivo and in vitro correlations. Magn. Reson. Med. 1994; 32(2): 157-163.

[19] Pantoni L, Garcia J. The significance of cerebral white matter abnormalities 100 years after Binswanger's Report. Stroke 1995;26(7): 1293-1301.

[20] Eichler FS, Itoh R, Barker PB, Mori S, Garrett ES, van Zijl PC, Moser HW, Raymond GV, Melhem ER. Proton MR spectroscopic and diffusion tensor brain MR imaging in X-linked adrenoleukodystrophy: initial experience. Radiology. 2002; 225: 245-252. 